\title{
Delimitation of service areas in reverse logistics networks with multiple depots
}

\author{
TRP Ramos ${ }^{1 *}$ and RC Oliveira ${ }^{2}$ \\ ${ }^{1}$ ISCTE Business School - Lisbon University Institute, Lisbon, Portugal; and ${ }^{2}$ CESUR - IST - Technical \\ University of Lisbon, Portugal
}

This paper describes a heuristic approach for the definition of service (or influence) areas of multiple depots in a reverse logistics network. This work is based on a case study: the recyclable waste collection system with 5 depots that covers seven municipalities in the Alentejo region, southern Portugal. The system optimization considers two objectives, related with economic and organizational issues: minimizing the variable costs (function of the travelled distances by the collection vehicles), and the pursuit of equity, aiming at minimizing the workload differences among depots. The goal of balancing workloads poses a problem not usually treated in the existing literature. This fact required the development of a new approach with innovative elements adjusted to the existing circumstances.

Journal of the Operational Research Society (2011) 62, 1198-1210. doi:10.1057/jors.2010.83

Published online 23 June 2010

Keywords: multiple depots; service areas; heuristics; reverse logistics; vehicle routing

\section{Introduction}

Reverse logistics is becoming increasingly important as recycling and environmental concerns gain significance. This new reality has a strong impact on supply chain design, since it no longer ends when the product is delivered to the final customer, but it now also includes used products return aiming at recovering some value.

The design of a recyclable waste collection network involves: (i) strategic decisions, such as on the number and location of depots, sorting waste stations and containers, or defining the size, type and capacity of the transport fleet; (ii) tactical decisions, for example establishing the service area for each depot; (iii) operational decisions, such as defining the collection routes. This paper aims to support tactical decisions, a decision level not so commonly addressed in the literature as the other two. Most frequently, the depots influence areas are established when, at the strategic decision level, the number and location of depots are defined. However, there are cases where, although the strategic level decisions have been taken beforehand, circumstances require that the current operations policy is revised, namely in what concerns depots service areas (re)configuration. This was the scenario posed by the case study addressed in this paper that involves the company responsible for the recyclable waste

\footnotetext{
*Correspondence: TRP Ramos, ISCTE Business School - Lisbon University Institute, Avenida das Forças Armadas, Edifício ISCTE, Lisbon, 1649-026, Portugal.

E-mail: tania.ramos@iscte.pt
}

collection network covering seven municipalities of the Alentejo region, southern Portugal.

This company (Ambilital) owns and operates 5 depots and 1622 recyclable waste containers (651 glass bins, 513 paper bins, and 448 plastic/metal bins). These containers are located in 663 different sites, and the collection is performed by a heterogeneous fleet of seven vehicles. Figure 1 shows the location of both containers and depots and the number of vehicles by depot.

This company needed to identify the set of containers that should be served by each depot, that is, to define the geographic limits of the influence area for each of the 5 depots. Besides being concerned with minimizing operations variable costs (based on the distances travelled by the vehicles), the company wanted to find a solution that promotes equity among depots, which can be interpret as minimizing the differences in workload among depots.

This case study triggered off the development of a model to define depots service areas in reverse logistics networks with multiple depots, where two types of factors are taken into account: an economic factor (minimization of the operations variable costs) and an organizational factor (minimization of the differences in workload among depots).

The goal of balancing workload among depots - essential in problems with multiple depots where human resources, although part of the same organization, are fixed at each depot - poses a new problem in face of the existing literature. This required the development of a new approach with innovative elements adjusted to the existing circumstances. 


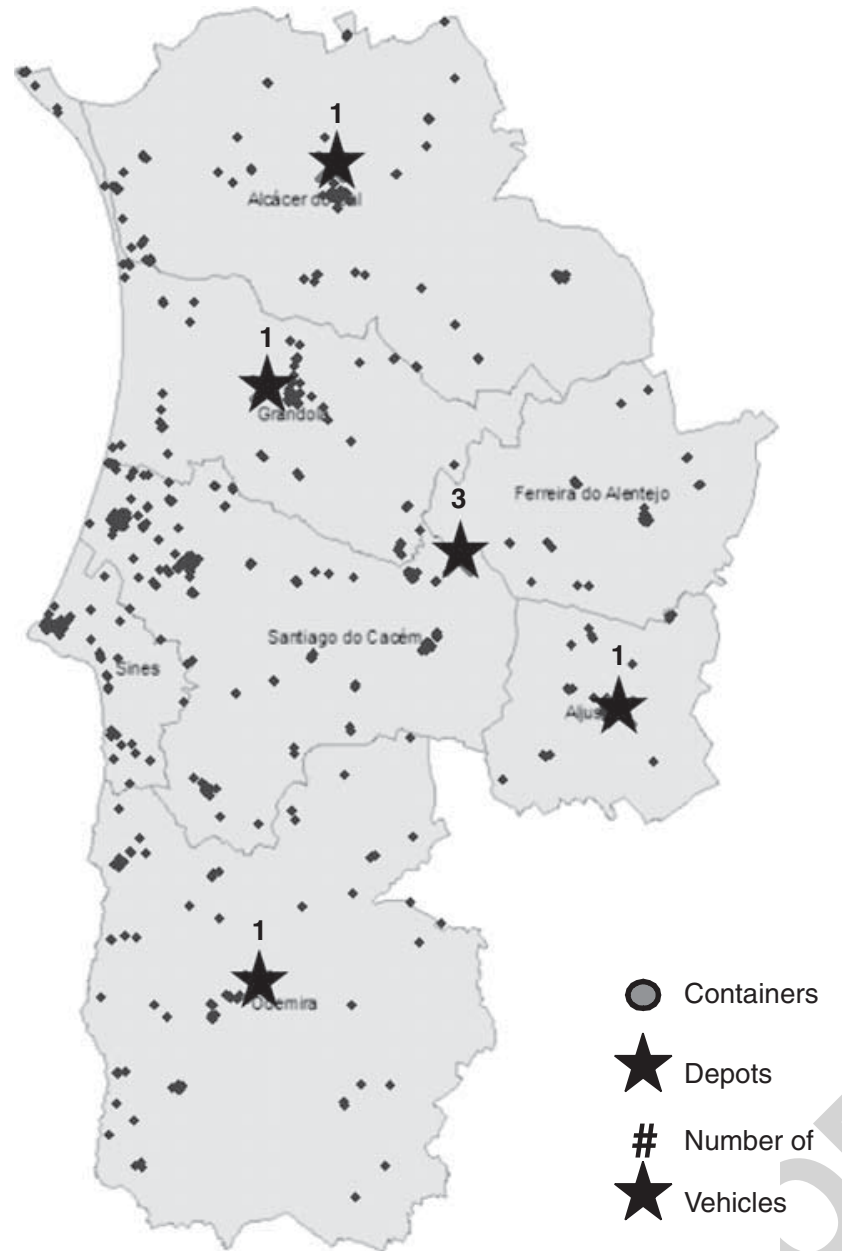

Figure 1 Location of the 1622 containers and the 5 depots and number of vehicles by depot.

Since the strategic level decisions (on the number and location of depots, vehicles and containers) had been taken beforehand, the problem of defining the depots influence areas (tactical decision level) can be approached in association with more operational issues related to establishing routes for the collection vehicles. From this viewpoint, in addition to having to determine the vehicle routes to collect a set of geographically dispersed containers, it is also necessary to decide:

- which containers are collected on each day (since the containers have different collection frequencies); and

- from which depot the collection of each container is performed.

Therefore, this problem adds two decision levels to the classical Vehicle Routing Problem, fitting the Multi-Depot Periodic Vehicle Routing Problem. This problem has seldom been studied in the literature, and consequently there are few models developed to solve it.
The depots multiplicity is the focus of this paper. The periodicity issue is covered in a simplified way: it was considered a 4 weeks' planning period, that corresponds to 20 workdays (since the lowest collection frequency found was monthly) and the collection frequency within containers of the same type of recyclable material was considered identical (but different among the three types of waste).

This paper is structured as follows. Following a brief review of the literature in 'Literature review' section, the proposed model for solving the problem is described in 'The algorithm' section. In 'Application to the case study' section, the computational results obtained for the case study are presented. Finally, conclusions are drawn and future work directions are discussed.

\section{Literature review}

The problem of defining service (or influence) areas appears in many circumstances and can be approached per se, that is, independently of other considerations or decision levels. That is, for instance, the case of districting, where a large region is partitioned into smaller sub-regions (districts), to facilitate the organization of the 'operations' that have to be performed within the region (Muyldermans et al, 2003). Districting problems arise in many real-world applications, such as political districting (Bozkaya et al, 2003), sales territories (Zoltners and Sinha, 1983), or waste collection (Mourão et al, 2009).

In multi-facility systems, such as logistics networks with multiple depots, influence (or service) areas are most commonly established at the strategic level, when the number and locations of these facilities are defined. Facility location models are widely covered in the literature. See Goetschalckx et al (2002), ReVelle and Eiselt (2005), or Melo et al (2009) for surveys about these models.

For logistics systems, depots influence areas are also established in connection with vehicle route definition, leading to the Multi-Depot Vehicle Routing Problem (MDVRP). There are few exact algorithms to solve this problem. Laporte et al (1984), as well as Laporte et al (1988) have developed exact branch and bound algorithms, but those are only applicable to small size instances. To solve large-scale instances, several heuristics have been developed.

Tillman and Cain (1972) present a savings method with a modified distance formula to include the existence of several depots. Golden et al (1977) have introduced the concept of borderline node, based on the calculation of the ratio proposed by Gillet and Johnson (1976) that relates the distance from each client to the nearest depot and the distance to the second nearest depot. If the value of this ratio is higher than a specific threshold, then the client is considered a borderline node.

Renaud et al (1996b) present a two-stage heuristic: (1) constructing an initial solution where each client is 
assigned to the nearest depot - and for each depot they apply the VRP heuristic 'Improved Petal' developed by Renaud et al (1996 a); (2) application of an algorithm (FIND), based on tabu search, to improve the initial solution found. Salhi and Sari (1997) have developed a heuristic for the multidepot vehicle fleet mix problem (MDVFM), and have also tested it on the MDVRP. The objective of this heuristic is simultaneously to build a set of routes and to determine the composition of the vehicle fleet at a minimum total cost (vehicle fixed cost plus mileage cost).

Lim and Wang (2005) proposed two methods to solve the multi-depot vehicle routing problem with fixed distribution of vehicles (MDVRPFD): two-stage and onestage approaches. The two-stage approach decomposes the MDVRPFD into two independent sub-problems (assignment and routing), and solves them separately. In the onestage approach, assignment and routing are solved in an integrated way.

Crevier et al (2007) study an extension of the MDVRP in which vehicles may be replenished at intermediate depots along their route. The authors propose a heuristic combining the adaptative memory principle and a tabu search method for the generation of a set of routes and integer programming in the execution of a set partitioning algorithm for the determination of the least cost feasible routes.

In face of this literature review, we can conclude that the models developed for the multi-depot problems do not consider the objective of balancing workload among depots. It was, therefore, necessary to develop a model that would include both the established objectives. This model is supported by heuristic procedures and follows an integrated approach between influence areas definition and routing.

However, it should be stressed that the key issue is the delimitation of the depots influence areas, for which workload balancing is a major concern (together with variable costs minimization). In order to estimate depot workloads, vehicle routes have to be designed, but within a simplified approach that avoids feeding in the model excessive details on daily operational conditions that would increase the model complexity (and computational burden) without significant impact at the tactical level decisions addressed here. These details are relevant for routes optimization at the operational decision level, but routes design in our model is simply a means to obtain reasonable estimates of depot workloads and routes travelling distances.

\section{The algorithm}

The definition of influence areas is done by assigning the collection sites to the 5 depots. It was, therefore, necessary to define an assignment criterion that would reflect the objectives we intend to reach - to minimize distances and to balance depots' workloads.

As the collection sites are scattered over the seven municipalities covered by the company, and they have different geographical positions in relation to the depots, the proposed algorithm uses different assignment criteria considering each specific positioning. To make this distinction, the concept of borderline node introduced by Golden et al (1977) was adapted as we want to classify the nodes in two categories regarding the two conflicting objectives: nodes that are much closer to one depot than to any other (and so they should be served from their closest depot in order to minimize the distance travelled) and nodes for which, given the proximity to more than one depot, there is no clear cut assignment (which can be used to balance the workload among depots). From the literature review, the concept of borderline node introduced by Golden et al (1977) was the most appropriate to accomplish these two objectives, fitting rather nicely the requirements of the problem. Following this approach, the collection sites are initially classified as borderline and non-borderline nodes. Depending on this initial classification, the moment of assignment to depots and the assignment criterion to be used are different, as will be specified ahead. The structure of the algorithm by modules is represented in Figure 2.

\section{Classification of the collection sites as borderline and non-borderline nodes}

The classification of the collection sites as borderline and non-borderline nodes is based on the ratio $r(i)$ proposed by Gillet and Johnson (1976). After computing the ratio, the value obtained is compared with a specific threshold

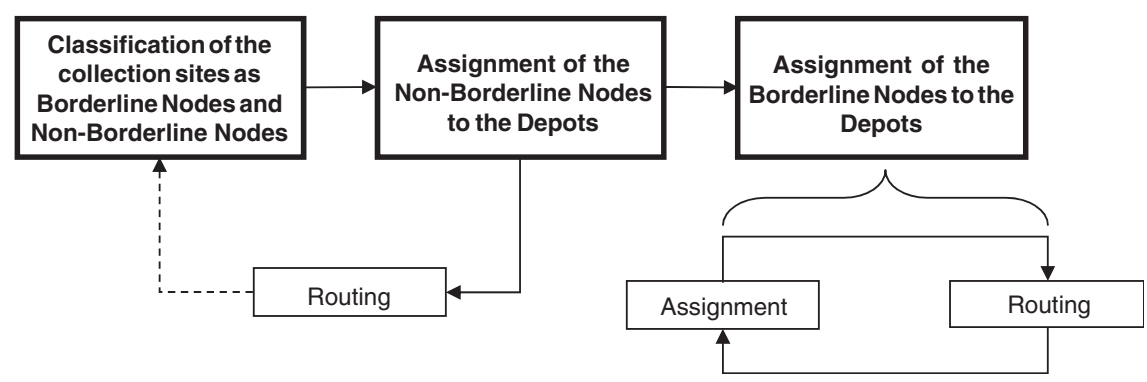

Figure 2 Algorithm structure by modules. 
For $\delta=0.7$ (e.g.)
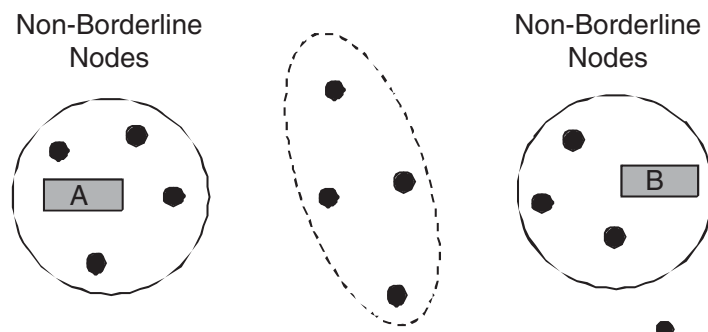

Nodes

Borderline Nodes

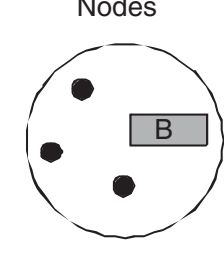

- Nodes

Depots

Figure 3 Borderline and non-borderline nodes representation.

defined a priori-parameter $\delta \in[0,1]$. If $r(i)<\delta$, then collection site $i$ is classified as non-borderline node; otherwise, the collection site $i$ is classified as borderline node. The concept of borderline node is represented in Figure 3.

For this specific case, two modifications to the ratio proposed by Gillet and Johnson (1976) were introduced:

- Transformed distance: since the total distance to be travelled includes the distance between the collection site and the depot (primary transport) and the distance between the depot and the sorting waste station (secondary transport) located in one of the depots, it is also necessary to consider for each collection site a part of the distance between the depot and the sorting waste station. Using the transformed distance, the assignment of the collection sites to the depot that is the sorting waste station is favoured, in order to avoid secondary transport. Thus, the distance between collection sites and depots to consider when calculating the ratio must be the transformed distance dis $^{\prime}{ }_{i d}$ :

$$
d i s_{i d}^{\prime}=d i s_{i d}+\beta \times d i s_{d T}
$$

where $d i s_{i d}$ represents the distance from collection site $i$ to depot $d ; d i s^{\prime}{ }_{d T}$ represents the distance from depot $d$ to the sorting waste station $T$; and $\beta$ represents cost ratio between secondary and primary transport.

- Index $j$ : considering that there are 5 depots, the index $j$ was introduced in the mentioned ratio, so that the ratio between the distance from collection site $i$ to the nearest depot and the distance from collection site $i$ to the $j$ th nearest depot ( $j$ varies from second to fifth) is calculated:

$$
r_{j}(i)=\frac{d i s_{i d 1}^{\prime}}{d i s_{i d j}^{\prime}}
$$

where $d i s^{\prime}{ }_{\mathrm{i} d 1}$ represents the distance from collection site $i$ to the nearest depot; and dis $^{\prime}{ }_{i d j}$ represents the distance from collection site $i$ to the $j$ th nearest depot.
To classify a collection site as borderline or nonborderline node, the result of the ratio with index $2\left(r_{2}(i)\right)$ is always used. However, when the collection site is considered a borderline node, it is relevant to know among which depots that node is considered 'borderline'. Therefore, we need to compare the ratios values with an index $j$ above 2 in order to assess if that collection site is a borderline node between more than two depots.

Parameter $\delta$ was also modified in this algorithm: instead of a single value, we admit that it may have different values for each pair of depots, originating a matrix $\delta_{d d}$. This adjustment was necessary because the borderline nodes are used to balance workload among depots, so there may be situations of unbalance between only two or more depots, with all the others being balanced. In this situation, the value of $\delta$ is changed for the corresponding pair(s) of unbalanced depot(s) in order to create more borderline nodes to balance the workload, while maintaining the previous $\delta$ values for the remaining depots.

The classification of collection sites as borderline or nonborderline node has a major impact on the algorithm performance and might influence the final solution, since the assignment criteria used for each category of nodes will be different, as described in the following sections. However, an iterative procedure described ahead adjusts parameters $\delta_{d d}$ so that the initial classification is revised and its influence upon the final solution is mitigated.

\section{Assignment of the non-borderline nodes to the depots}

Non-borderline nodes are much closer to one depot than to any other, so they should be served from their closest depot in order to minimize the distance to be travelled in the collection routes. However, the nearest depot may not have available capacity to collect all the non-borderline nodes assigned to it. In this situation, the question of which nodes should be left out of this initial assignment is posed.

To address this last question, an assignment urgency was calculated for each non-borderline node. The urgency is a way to define a precedence relationship between collection sites, determining the order in which collection sites should be assigned to depots. Potvin and Rousseau (1993) proposed methods to calculate the assignment urgency of the nodes to the vehicles in vehicle routing problem with time windows (VRPTW), and Giosa et al (2002) adapt these formulas for the MDVRP. For this case we used the calculation of the Simplified Assignment Urgency with the following formula:

$$
\mu_{i}=d i s_{i d 2}^{\prime}-d i s_{i d 1}^{\prime}
$$

where dis $^{\prime}{ }_{i d 2}$ represents the transformed distance between node $i$ and its second closest depot; and $d i s^{\prime}{ }_{i d 1}$ represents the transformed distance between node $i$ and its closest depot.

If the difference between the distance to the closest depot and the distance to the second closest depot is large, then 


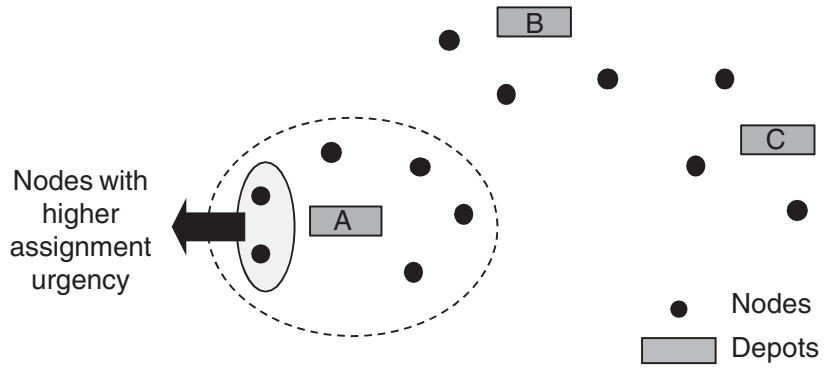

Figure 4 Assignment urgency representation.

the assignment urgency of that node is high too. A high value of $\mu_{i}$ means that it is more convenient to assign the node $i$ to its closest depot than assigning it to any other depot. The concept of assignment urgency is represented in Figure 4.

To determine whether a depot has capacity to serve all the non-borderline nodes initially assigned to it, it is necessary to define routes in order to compute the time spent to collect them.

If the number of hours necessary to collect all the nonborderline nodes is larger than the number of working hours available at the depot, then it is necessary to remove nodes from that depot. To remove a node from a depot, the algorithm places a $M$ value (very high value) on the transformed distance between that node and the depot, in order to prevent that node from being again assigned to that depot that has no capacity to collect it. When removing the node from the depot, we need to verify if it is still considered a non-borderline node, or if it becomes a borderline node. For that, the algorithm goes back to the beginning, but using as an input the transformed distance matrix updated by the introduction of the $M$ value. This procedure is made node by node, starting at the end of a list ranked by assignment urgency, until the depot has enough capacity to serve all the non-borderline nodes assigned to it. When this situation is reached, the algorithm moves on to the next depot, until all the non-borderline nodes are assigned to a depot.

\section{Assignment of the borderline nodes to the depots}

Borderline nodes are those for which there is uncertainty regarding their assignment, given the proximity to more than one depot. For this reason, and considering that the aim of this work is to find a compromise solution between minimizing distance and balancing workload among depots, borderline nodes will be used to balance depots workload since the previous assignment of the nonborderline nodes merely considered the distance minimization objective.

The decision to assign a borderline node to a depot is based on an indicator developed to measure the attractiveness of the depots for each borderline node. This attractiveness measure reflects the concern with balancing workload among depots-without overlooking distance minimization - indicating which depot is more attractive for each borderline node.

Considering:

$D_{i} \quad$ set of depots for which node $i$ is considered 'borderline'

$d i s_{i r_{d}}$ distance between node $i$ and the nearest route $r$ belonging to depot $d$

$C T_{d} \quad$ workload of depot $d$

$V_{d} \quad$ number of vehicles at depot $d$

$M_{i d} \quad$ attractiveness measure of depot $d$ to node $i$

$\alpha \quad$ weight of the distance between node $i$ and the nearest route $r$ belonging to depot $d$ in the attractiveness measure

Then:

$$
\begin{aligned}
M_{i d}= & 1 \\
& -\left[\alpha \times \frac{d i s_{i r_{d}}}{\sum_{r_{d} \in D_{i}} d i s_{i r_{d}}}+(1-\alpha) \times \frac{C T_{d} / V_{d}}{\sum_{d \in D_{i}} C T_{d} / V_{d}}\right]
\end{aligned}
$$

This attractiveness measure considers that there are already routes defined (although provisional) that may be expanded to include the borderline nodes.

For each borderline node $i$, as many $M_{i d}$ as the dimension of $D_{i}$ set are calculated. The depot that has the highest value is the more attractive to collect node $i$, and consequently node $i$ is assigned to that depot.

After computing the attractiveness measure $M_{i d}$ for all borderline nodes, the nodes are then assigned one at a time. This procedure allows to assess the impact on depot workload after each assignment and to recalculate the attractiveness measures considering the new assigned node. This procedure is more time consuming than assigning all nodes at once and to evaluate depot workload afterwards. However, the quality of the solution in terms of balancing workload among depots would be worse, since the calculation of the measure $M_{i d}$ would only consider the workload resulting from the collection of the non-borderline nodes, and would not be updated as new nodes were assigned.

Since the objective is to balance workload by inserting the borderline nodes in the collection routes, then we must start by assigning borderline nodes to the depot with the lowest workload at that moment. Then, it is necessary to check if there are borderline nodes that, according to the $M_{i d}$ value, should be assigned to that depot. If there are not, then the algorithm performs the same procedure for the second depot with the lowest workload, and so on, until it finds borderline nodes that, according to the $M_{i d}$ value, are assigned to that depot.

Since assignment will be performed node by node, we have tested five heuristic rules to decide which will be the 
first node to assign, among all the nodes that could be assigned to that depot:

1. Assign first the node with the highest workload (measure in hours needed to collect that node)

$$
C T_{i}=\sum_{m \in M} C R_{i m}\left(t c+\frac{d i s_{c}}{V L}\right)
$$

where $C T_{i}$ represents the workload of collection site $i$; $M$ represents the set of recyclable materials \{glass, paper, plastic/metal $\} ; C R_{\text {im }}$ represents the number of $m$ containers in collection site $i$; $t c$ represents the time required to collect a container; $d i s_{c}$ represents the distance between containers; and $V L$ represents the vehicle average speed within localities $(\mathrm{km} / \mathrm{h})$.

2. Assign first the node with the highest contribution for balancing workload versus contribution to distance increase, Cont ${ }_{i}$, defined as:

$$
\text { Cont }_{i}=\frac{C T_{i} / C T_{d}}{\left(2 \times d i s_{d}\right) / d i s_{d}}
$$

where $C T_{i}$ represents the workload of collection site $i$; $C T_{d}$ represents the workload of depot $d$; dis $s_{i d}$ represents the distance between collection site $i$ and depot $d$; and $d i s_{d}$ represents the total distance to be travelled from $\operatorname{depot} d$.

3. Assign first the node with the highest assignment urgency (parallel assignment urgency).

$$
\mu_{i}=\sum_{d \in D_{i}}\left(d i s_{i d}^{\prime}-d i s_{i d w}^{\prime}\right)
$$

where $\mu_{i}$ represents the assignment urgency of collection site $i ; D_{i}$ represents the set of depots for which collection site $i$ is considered 'borderline'; dis' ${ }_{i d}^{\prime}$ represents the transformed distance between collection site $i$ and depot $d$; and $d i s_{i d w}^{\prime}$ represents the transformed distance between collection site $i$ and the depot with the lowest workload.

4. Assign first the node closer to the nodes already assigned to that depot;

5. Assign first the node with the highest $M_{i d}$ value.

Rules 1 and 2 presented better results in terms of workload balance, distance travelled and influence areas continuity. The results presented in 'Application to the case study' section were obtained applying rule 1 .

\section{Routing}

Routing impacts on service areas definition, and service areas definition impacts on routing: these are interrelated decisions. Namely, the depots workload is obtained from the routes durations, so a route design procedure had to be included in the model. The savings algorithm (Clarke and Wright, 1964) was adopted due to its simplicity and effectiveness in providing reasonable estimates of the workload posed on each depot. The routing procedure is executed many times within the algorithm and it is the more time consuming module, and therefore computational efficiency is a critical issue. More sophisticated routing procedures might place a too heavy computational burden, whereas strict route optimization is not a key concern here, as discussed before, since all that is required is reasonable estimates of depots workloads and route distances. The savings algorithm proved to be an effective method to provide these approximations.

The routes are defined by recyclable waste type and by vehicle from each depot. Each type of recyclable material must be collected separately. The routes are periodically repeated along the planning horizon according to the collection frequency for each waste type. Joining the routes of the three types of waste, we obtain the depot routes for the planning period of 20 workdays.

\section{Final iterative procedure}

After a solution is obtained, we need to analyze if that solution is satisfactory in terms of distance and workload difference among depots, since parameters $\delta_{d d}$ and $\alpha$ may be adjusted in order to produce more satisfactory solutions. The analysis of the solution obtained and the parameter adjustment are then performed in order to generate diversified alternative solutions.

According to the results obtained, it might be necessary to perform the following adjustments:

- If the difference between depots workloads is higher than a maximum value:

- decrease parameter $\delta_{d d}$, so that there will be more borderline nodes;

o decrease parameter $\alpha$, so that the depot's workload has more influence on the assignment of the borderline nodes.

- If total distance travelled is higher than a maximum value:

- increase parameter $\delta_{d d}$, so that more nodes are assigned according to the proximity distance criterion (there will be more non-borderline nodes);

- increase parameter $\alpha$, so that distance has more influence on the assignment of the borderline nodes.

After parameters adjustment, a new iteration is performed, producing a new solution. Parameters $\delta_{d d}$ and $\alpha$ were adjusted with one decimal variation in each 
iteration. For this case, the maximum value for distance travelled was set to $10 \%$ plus the minimum distance found and the maximum value for the difference between depots workloads was set to $40 \%$. When a solution fits both criteria, this final iterative procedure stops.

\section{Application to the case study}

To reduce the problem size, the 663 collection sites were grouped in 212 clusters, corresponding to localities or isolated locations. According to the analysis of the existing geographical maps, we verified that the routes within each cluster will not be affected, in terms of distance and time, by how the visit to that location is inserted in the vehicle routes. The distance travelled within the locality remains practically unchanged no matter what the actual entry or exit points are; hence it is practicable to treat the various containers to collect within any locality (cluster) as a single node in a wider route. The time spent and distances travelled within each cluster were modelled as a function of the number of containers in that locality. It should be stressed that problem size reduction is not necessary, but merely convenient since it reduces the computational burden. Furthermore, and from an operational point of view, once a vehicle visits one cluster (locality), all the containers within that locality should be collected since this is more efficient than having different vehicles visiting the locality (as long as joint collection is feasible, which is the case in our case study).

The developed model has three parameters, so the results obtained depend on the value defined for each one of them. The value of parameter $\beta$ was set at 0.25 for this particular case study, based on an analysis of relations between typical distances and costs associated with the collection routes and secondary transport. Regarding parameters $\alpha$ and $\delta_{d d}$, it is not possible to define a priori which values should be adopted.

In the process of balancing service areas, parameters $\alpha$ and $\delta_{d d}$ are adjusted as the algorithm produces solutions. Some previous simulations were performed in order to observe how the algorithm reacted to changes in those parameters. To illustrate how the algorithm performs, the results obtained with (all $\delta_{d d}=0.5$ and $\alpha=0$ ) and with (all $\delta_{d d}=1$ and $\left.\alpha=1\right)$ are presented. In the first case, $80 \%$ of the nodes were classified as borderline nodes, and their assignment depends solely on the workload of the depots $(\alpha=0$ means that the weight of the distance in the attractiveness of a depot is null). The results obtained can be seen in Figure 5. As expected, the resulting service areas lead to a balanced solution in terms of the working hours of the 5 depots, but with a very high geographical discontinuity that increase the total distance to be travelled. Besides the value of the parameters used, the dispersion

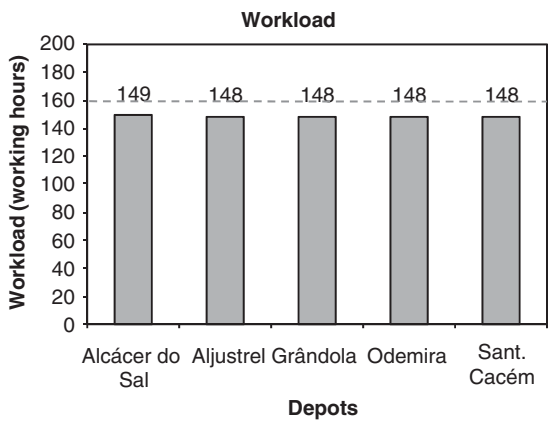

Workload difference between depots ((maximum workload/minimum workload -1 ) $\times 100): 1 \%$

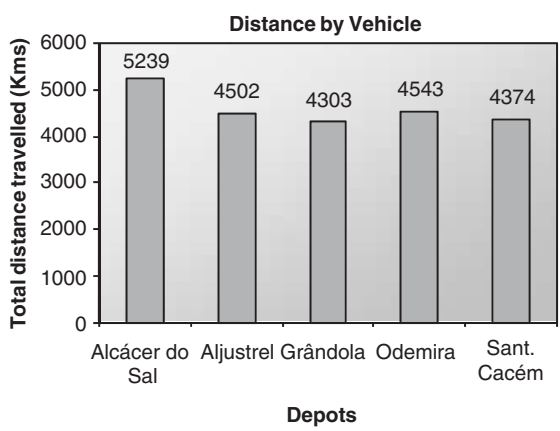

Total distance travelled: $31708 \mathrm{Kms}$
X Collection Sites assigned to Alcácer do Sal (Depot 1)

- Collection Sites assigned to Aljustrel (Depot 2)

$\Delta$ Collection Sites assigned to Grândola (Depot 3)

+ Collection Sites assigned to Odemira (Depot 4)

- Collection Sites assigned to Santiago do Cacém (Depot 5)

Depot

Figure 5 Solution obtained with $\delta_{d d}=0.5$ and $\alpha=0$. 
obtained also derives from the constructive character of the heuristic, which assigns first the borderline nodes with a higher workload as a way to promote a balance in the working hours of the depots.

In the second case, all nodes are classified as nonborderline nodes (due to $\delta_{d d}=1$ ), so their assignment depends solely on the transformed distance to the depot. The results obtained can be found in Figure 6, where we can observe unbalanced working hours among the 5 depots, but a nearly $30 \%$ reduction in terms of the distance to be travelled.

The iterative process of defining the service areas starts with the solution with the lowest total distance travelled found, and then decreases parameters $\delta_{d d}$ and $\alpha$ until a balanced solution concerning depots workload is found. The preliminary simulations performed served to set the initial values for parameters $\delta_{d d}$ and $\alpha$ and the corresponding solution with the minimal total distance travelled found is represented in Figure 7(a). Observing the depot's workload for this solution, we verified a higher unbalance between the loads of depot 2 and the remaining, as well as between depot 1 and the others. To achieve a more balanced solution, parameters $\delta_{d d}$ and $\alpha$ were adjusted with a one decimal variation in each iteration. So, for each unbalanced pair of depots, the value of $\delta_{d d}$ was decreased until we find a more satisfactory solution for both criteria; the value of parameter $\alpha$ was decreased and increased until the stopping criterion is reached.

Seven main iterations were performed by altering parameters $\delta_{d d}$ and $\alpha$. Table 1 shows details of each iteration and their performance is represented in Figure 8, where the Pareto border is defined.

Comparing the solution of iteration 7 (represented in Figure 7(b)) with the initial solution, we notice an increase in the total distance to be travelled of only $3.7 \%$, but the unbalance of workloads, measured by the difference between the maximum and the minimum workload, decreases by $77 \%$.

To support the decision-maker in selecting the solution that should be adopted, the solutions obtained must be globally evaluated and compared. Since there are two evaluation criteria, we used an additive model to compute an overall score for each solution, where the weight $\lambda$ represents the relative importance given to the Distance criterion, or more precisely, to the swing associated to its range scale:

$$
\begin{aligned}
\text { Global Score }= & \lambda f(\text { Distance }) \\
& +(1-\lambda) g(\text { Workload difference })
\end{aligned}
$$
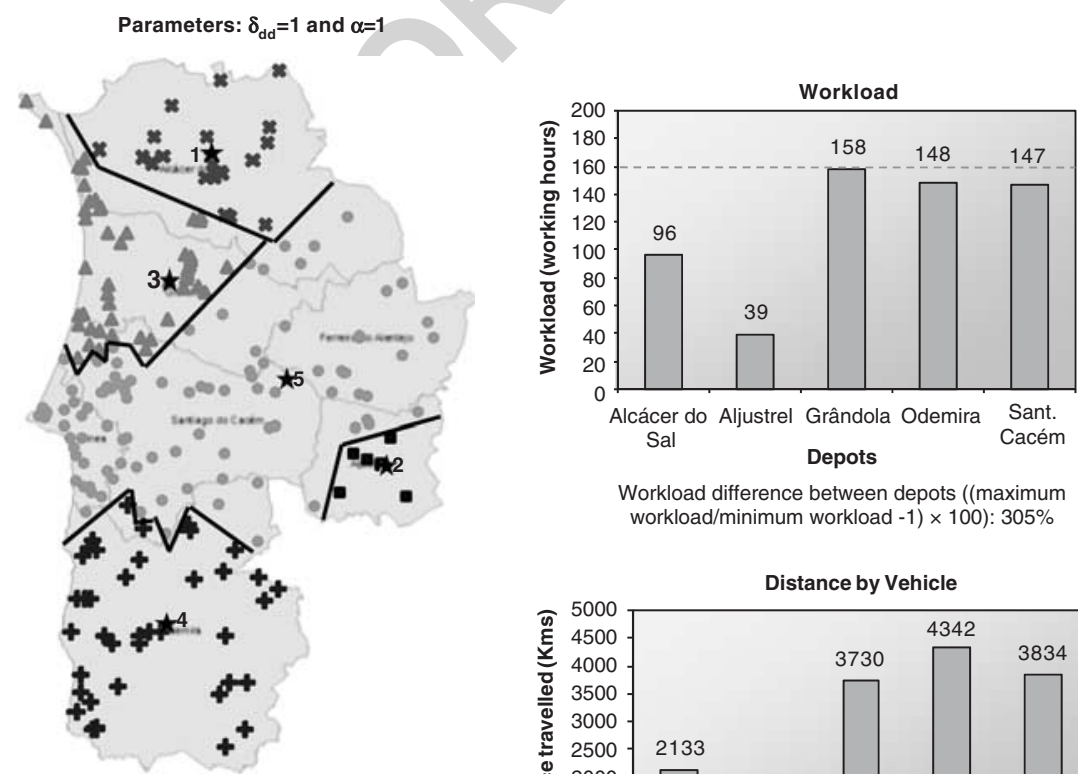
workload/minimum workload -1) $\times 100): 305 \%$

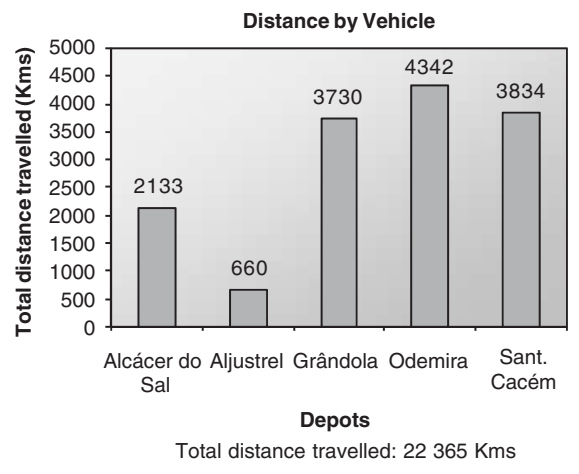

\footnotetext{
Collection Sites assigned to Alcácer do Sal (Depot 1)

- Collection Sites assigned to Aljustrel (Depot 2)

$\Delta$ Collection Sites assigned to Grândola (Depot 3)

+ Collection Sites assigned to Odemira (Depot 4)

Collection Sites assigned to Santiago do Cacém (Depot 5)

$\star$ Depot
}

Figure 6 Solution obtained with $\delta_{d d}=1$ and $\alpha=1$. 
a

\begin{tabular}{|c|c|c|c|c|c|}
\hline \multirow{2}{*}{\multicolumn{2}{|c|}{$\delta_{\text {dd } 1}$}} & \multicolumn{4}{|c|}{ Initial Solution } \\
\hline & & 2 & Dеро & & \\
\hline \multirow{4}{*}{ 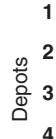 } & - & 0.6 & 0.7 & 0.7 & 0.7 \\
\hline & & & 0.6 & 0.6 & 0.6 \\
\hline & & & - & 0.7 & 0.7 \\
\hline & & & & - & 0.7 \\
\hline & & & & & - \\
\hline
\end{tabular}

$\alpha=0.8$

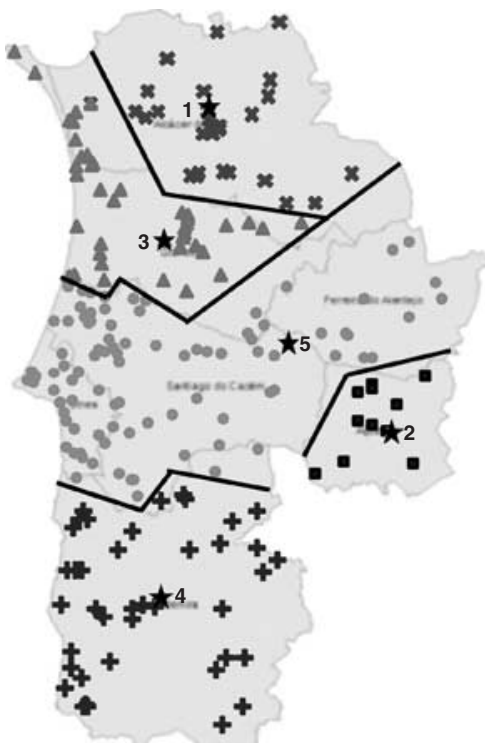

× Collection Sites assigned to Alcácer do Sal (Depot 1)

Collection Sites assigned to Aljustrel (Depot 2)

$\Delta$ Collection Sites assigned to Grândola (Depot 3)

+ Collection Sites assigned to Odemira (Depot 4)

- Collection Sites assigned to Santiago do Cacém (Depot 5)

$\uparrow$ Depots

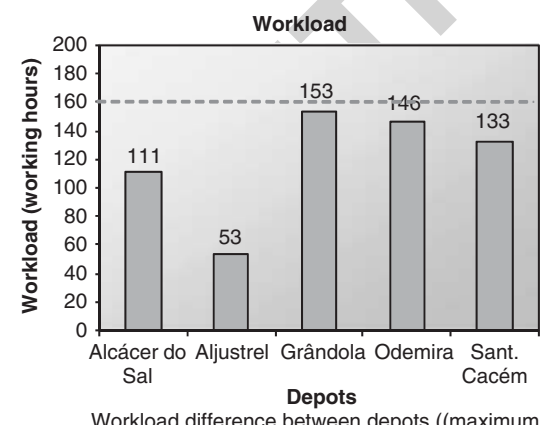

Distance by Vehicle

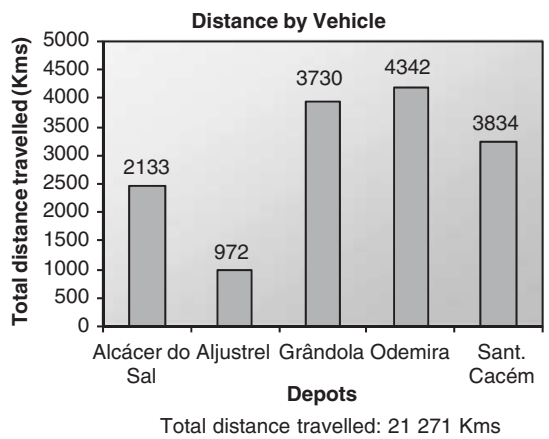

b Iteration 7 Solution

\begin{tabular}{|c|c|c|c|c|c|}
\hline \multirow{2}{*}{$\delta_{\text {dd }}$} & \multicolumn{5}{|c|}{ Depots } \\
\hline & 1 & 2 & 3 & 4 & 5 \\
\hline \multirow{4}{*}{$\begin{array}{l}\frac{n}{0} \\
\frac{0}{0} \\
\stackrel{0}{0}\end{array}$} & - & 0.5 & 0.6 & 0.7 & 0.7 \\
\hline & & - & 0.2 & 0.2 & 0.3 \\
\hline & & & - & 0.7 & 0.7 \\
\hline & & & & - & 0.7 \\
\hline 5 & & & & & - \\
\hline
\end{tabular}

$\alpha=0.6$

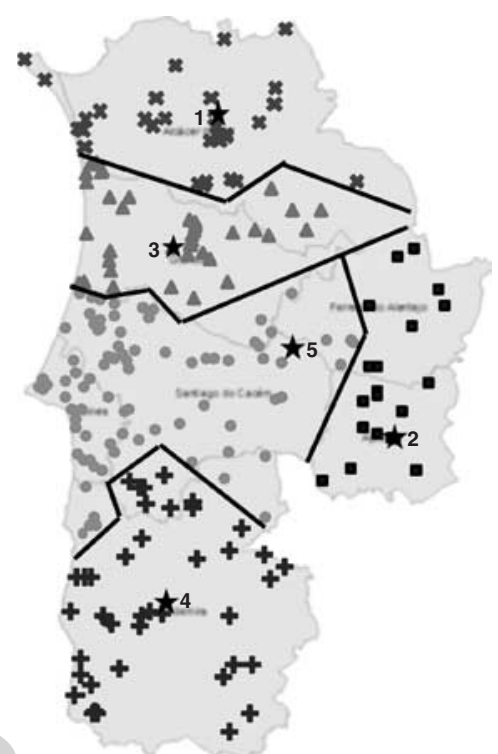

Collection Sites assigned to Alcácer do Sal (Depot 1)

- Collection Sites assigned to Aljustrel (Depot 2)

Collection Sites assigned to Grândola (Depot 3)

+ Collection Sites assigned to Odemira (Depot 4)

- Collection Sites assigned to Santiago do Cacém (Depot 5)

$\star$ Depots

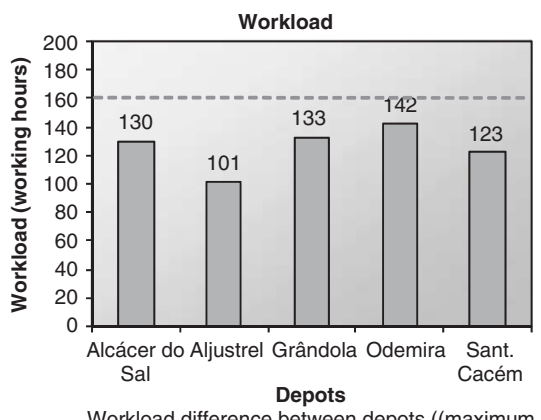

Workload difference between depots ((maximum workload/minimum workload -1) ×100): $40 \%$

Distance by Vehicle

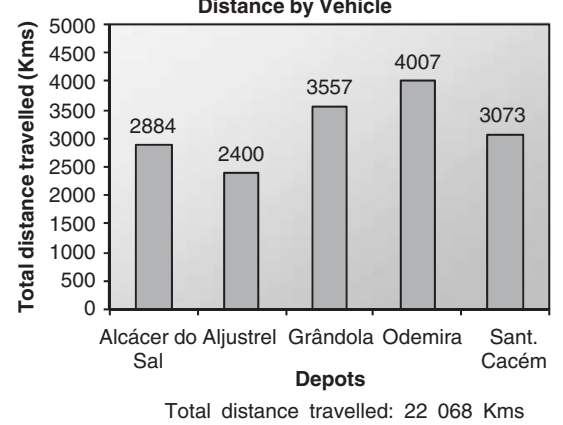

Figure 7 Initial solution (a) versus iteration 7 solution (b). 


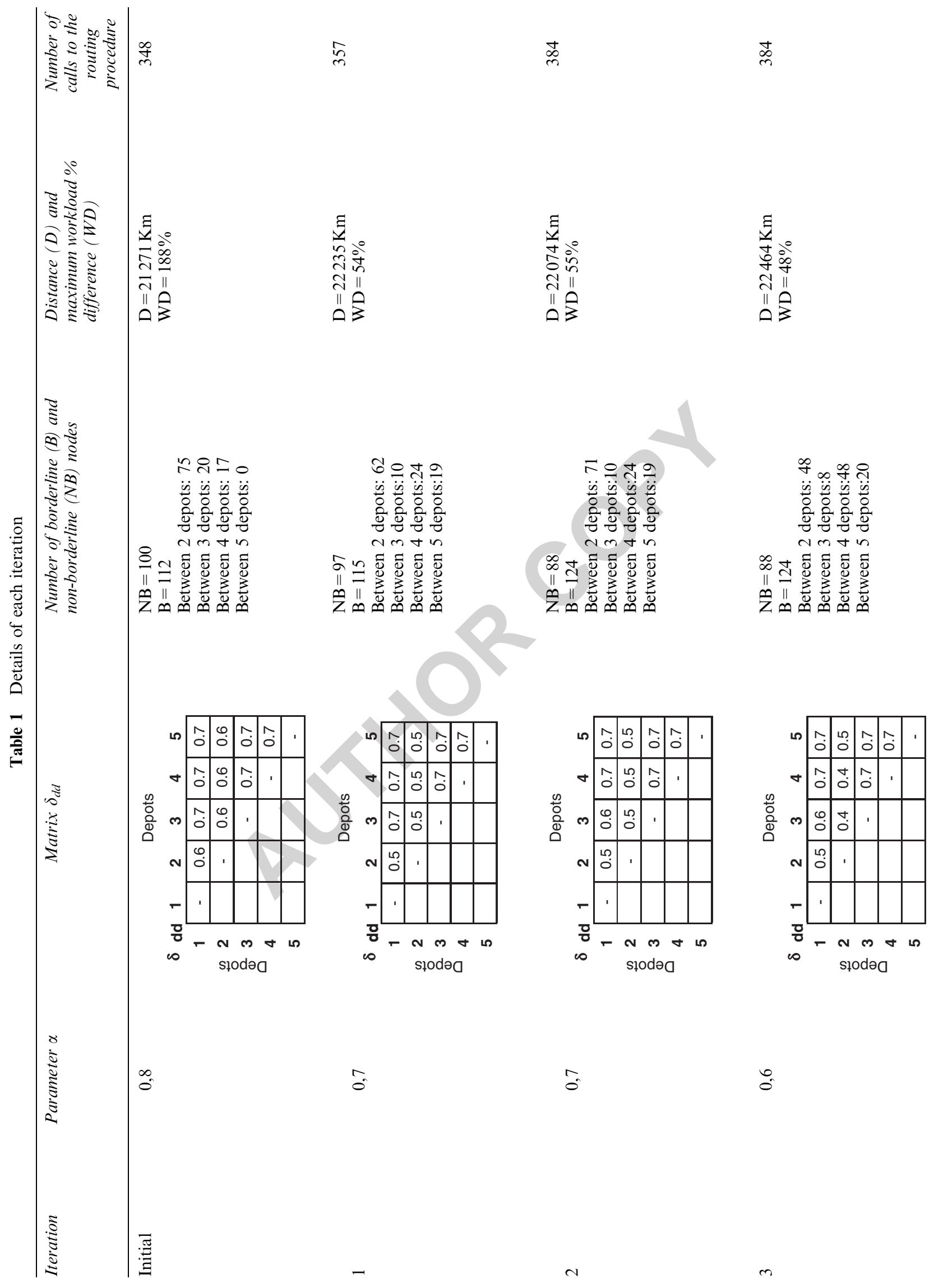




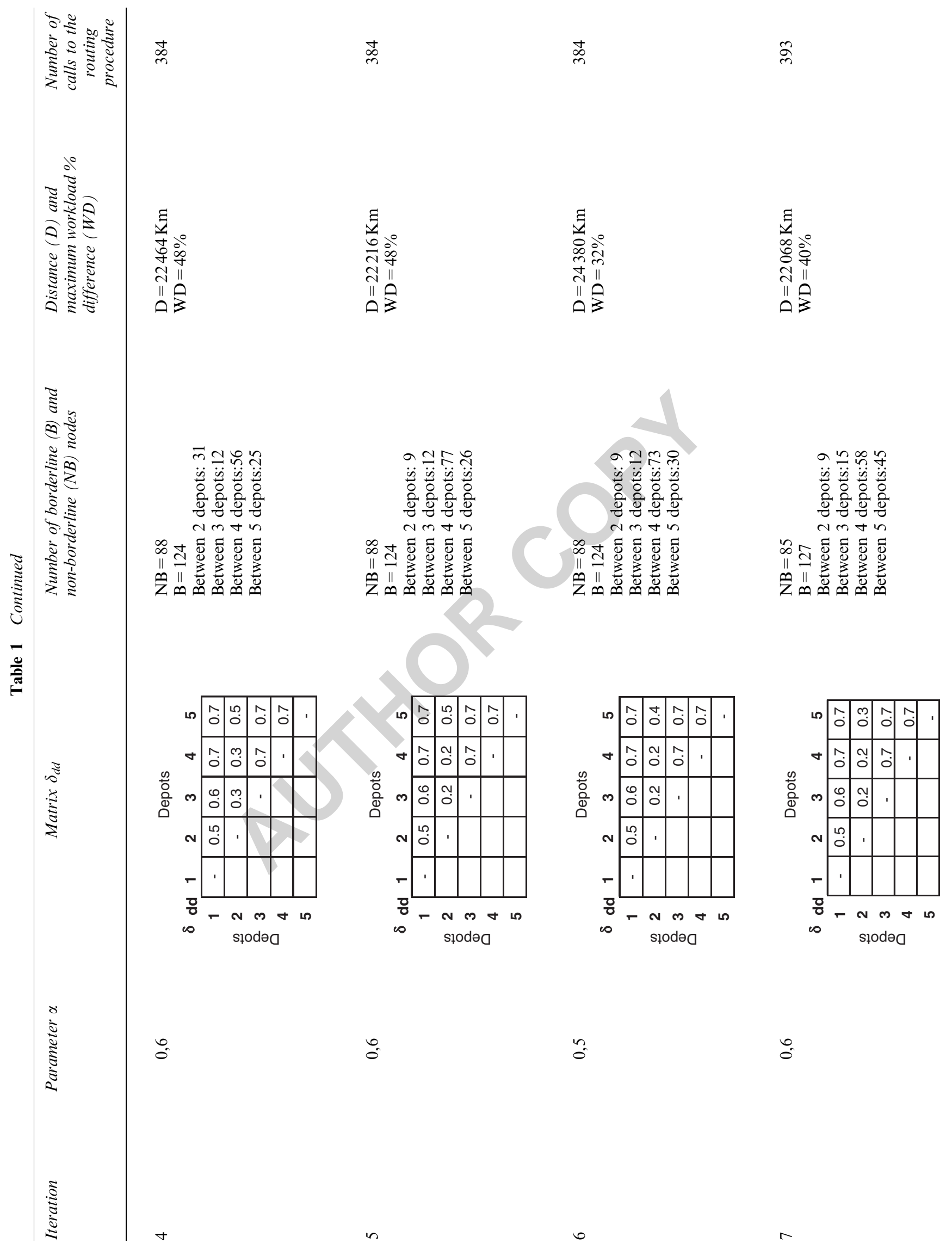




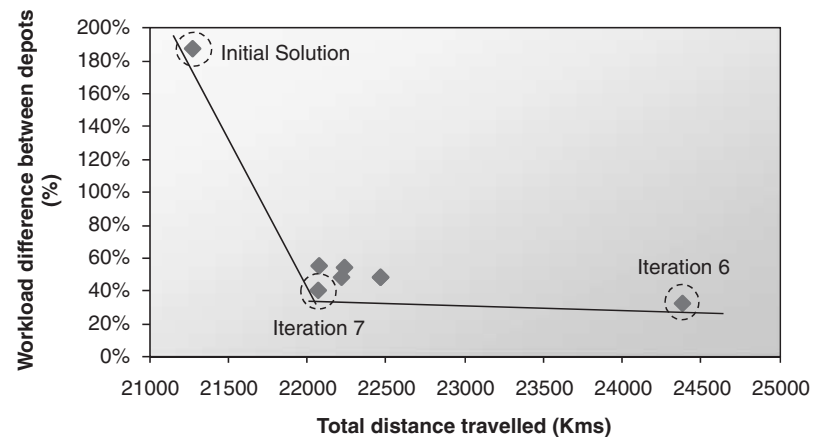

Figure 8 Solutions' performances on both objectives.

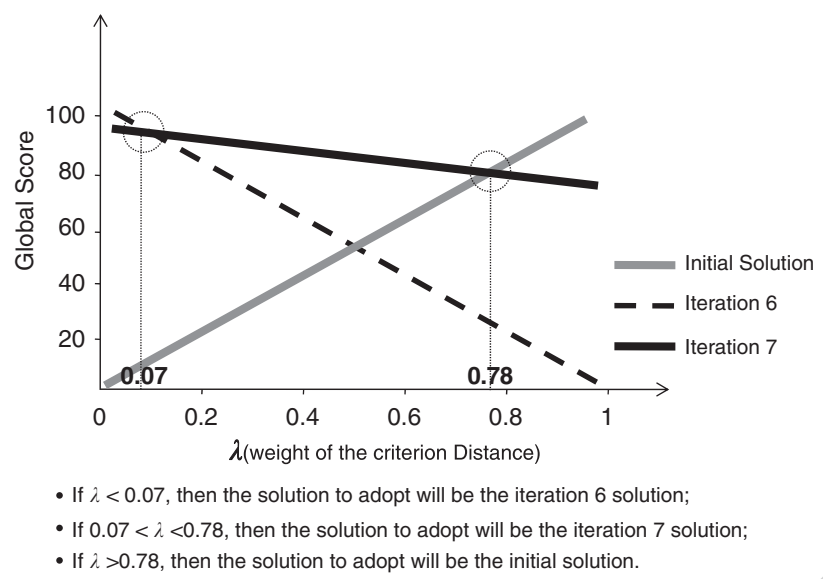

Figure 9 Sensitivity analysis on the weight of the criterion Distance $(\lambda)$.

where $f(D)$ and $g(W)$ represent the value functions for the Distance (D) and the Workload difference (W) criteria, respectively. These value functions were assumed to be linear, ranging from 0 (worst performance on the criterion, for example maximum travelling distance) to 100 (best performance).

The global score of each iteration depends on the weight $\lambda$. A sensitivity analysis on the weight of the Distance criterion is shown in Figure 9, which allows the decisionmaker to see how a change in this weight would affect the overall score of the various solutions.

\section{Conclusions}

The problem of delimiting the influence areas of 5 depots belonging to a solid recyclable waste collection system in Alentejo, Portugal was studied. Two conflicting objectives were present: variable cost minimization (related to distance travelled by vehicles) and equity promotion (related to workload balance among depots). This is not tackled by existing models in the literature and led to the development of a heuristic approach with innovative elements adjusted to these circumstances.
The definition of the service areas (an assignment problem) cannot be analyzed in isolation from the routing problem, given the existing interdependence: routes definition both influences and it is influenced by the service areas covered by each depot. Therefore, the model developed has followed an integrated approach between assignment of collection sites to depots (defining influence areas) and routing through an iterative procedure.

The model explores the concept of a borderline node (between two or more depots) which is defined by parameter $\delta_{d d}$. The final assignment of the borderline nodes results from an iterative process where an indicator that measures the attractiveness of the depots, and a route construction algorithm are applied.

The 'compromise level' between distance minimization and workload balancing has to be defined by the decisionmaker, with that 'level' being mirrored in the value of the parameters of the model. The impact of the model parameters was illustrated in this specific case.

To reach a compromise solution between the two objectives, the methodology used consisted in finding a solution with the lowest total distance travelled, and then adjusting parameters $\alpha$ and $\delta_{d d}$ to find a more workload balanced solution. As expected, the solution with the lowest total distance travelled corresponds to a very unbalanced solution regarding the workload of each depot. This derives in part from poor (previous) decisions on the location of the depots. To improve the balance of that solution, decreasing $\alpha$ and the parameter $\delta_{d d}$ between the more unbalanced pairs of depots has proven to be very effective in reaching this balance, not causing a significant increase in the total travelled distance.

We have also demonstrated that, in this case, using only the distance to the nearest depot as an assignment criterion (setting parameter $\delta_{d d}=1$ ) does not produce necessarily good solutions concerning the minimization of distance to be travelled. The assignment of all borderline nodes depends on the routes already built for each depot, which produces a better solution regarding the distance minimization criterion.

The proposed model has potential to be improved in future research and this is briefly discussed herein.

The model can be enhanced to solve problems where different collection frequencies for the sites are considered. Thus, both multiplicity and periodicity issues will be well thought-out.

To improve the results obtained, a local search procedure can be included in the model. Some operators could be executed: removal of collection sites from their current depot and assigning them to another depot, removal of collection sites from their routes and reinserting them into different routes (belonging to the some depot), or changing a collection site position within a route. A meta-heuristic, for example tabu search, could be applied to produce better results. 
Finally, another interesting research direction is embracing some strategic decisions in logistic network design. We focused our work in tactical decisions, where the number and location of the depots were an input data. Towards a global system optimization, the number and location of depots should be decision variables instead of constraints. In this case, we will deal with a different type of problema multi depot location routing problem.

\section{References}

Bozkaya B, Erkut E and Laporte G (2003). A tabu search heuristic and adaptive memory procedure for political districting. Eur $J$ Opl Res 144: 12-26.

Clarke G and Wright JW (1964). Scheduling of vehicles from a central depot to a number of delivery points. Opns Res 12: 519-653.

Crevier B, Cordeau JF and Laporte G (2007). The multi-depot vehicle routing problem with inter-depot routes. Eur J Opl Res 176: $756-773$.

Gillet B and Johnson J (1976). Multi-terminal vehicle-dispatch algorithm. Omega 4: 711-718.

Giosa I, Tansini I and Viera I (2002). New assignment algorithms for the multi-depot vehicle routing problem. J Opl Res Soc $\mathbf{5 3}$ : 997-984.

Goetschalckx M, Vidal CJ and Dogan K (2002). Modeling and design of global logistics systems: A review of integrated strategic and tactical models and design algorithms. Eur J Opl Res 143 : $1-18$.

Golden BL, Magnanti TL and Nguyen HQ (1977). Implementing vehicle routing algorithms. Networks 7: 113-148.

Laporte G, Nobert Y and Arpin D (1984). Optimal solutions to capacitated multi-depot vehicle routing problems. Congr Numer 44: $283-292$.
Laporte G, Nobert Y and Taillefer S (1988). Solving a family of multi-depot vehicle routing and location-routing problems. Transport Sci 22: 161-172.

Lim A and Wang F (2005). Multi-depot vehicle routing problem: A one-stage approach. IEEE Trans Autom Sci Eng 2: 397-402.

Melo MT, Nickel S and Saldanha-da-Gama F (2009). Facility location and supply chain management-A review. Eur J Opl Res 196: 401-412.

Mourão MC, Nunes AC and Prins C (2009). Heuristic methods for the sectoring arc routing problem. Eur J Opl Res 196: $856-868$.

Muyldermans L, Cattrysse D and Van Oudheusden D (2003). District design for arc-routing applications. J Opl Res Soc 54: 1209-1221.

Potvin J and Rousseau J (1993). A parallel route building algorithm for the vehicle routing and scheduling problem with time windows. Eur J Opl Res 66: 331-340.

Renaud J, Boctor FF and Laporte G (1996a). An improved petal heuristic for the vehicle routing problem. J Opl Res Soc 47: 329-336.

Renaud J, Laporte G and Boctor FF (1996b). A tabu search heuristic for the multi-depot vehicle routing problem. Comput Opl Res 23: 229-235.

ReVelle CS and Eiselt HA (2005). Location analysis: A synthesis and survey. Eur J Opl Res 165: 1-19.

Salhi S and Sari M (1997). A multi-level composite heuristic for the multi-depot vehicle fleet mix problem. Eur J Opl Res 103: 95-112.

Tillman FA and Cain TM (1972). An upperbound algorithm for the single and multiple terminal delivery problem. Mngt Sci 18: 664-682.

Zoltners AA and Sinha P (1983). Sales territory alignment-A review and model. Mngt Sci 29: 1237-1256.

Received December 2009; accepted April 2010 after one revision 